\title{
Mixed mode oscillations in the Bonhoeffer-van der Pol oscillator with weak periodic perturbation
}

\author{
Ekaterina Kutafina
}

Received: 19 November 2013 / Accepted: 2 December 2013 / Published online: 8 January 2014

(C) The Author(s) 2013. This article is published with open access at Springerlink.com

\begin{abstract}
Following the paper of Shimizu et al. (Phys Lett A 375:1566, 2011), we consider the Bonhoeffer-van der Pol oscillator with non-autonomous periodic perturbation. We show that the presence of mixed mode oscillations reported in that paper can be explained using the geometric singular perturbation theory. The considered model can be re-written as a fourdimensional (locally three-dimensional) autonomous system, which under certain conditions has a folded saddle-node singularity and additionally can be treated as a three time scale one.
\end{abstract}

Keywords Folded saddle-node - Three time scale system - Mixed mode oscillation . Singular perturbation $\cdot$ Canard

Mathematical Subject Classification (2000) $\quad 34 \mathrm{C} 26 \cdot 34 \mathrm{E} 13 \cdot 34 \mathrm{E} 17 \cdot 37 \mathrm{C} 60 \cdot 94 \mathrm{C} 99$

\section{Introduction}

There exist a lot of different mechanisms which can create a mixed mode oscillations (MMOs) in multiple time scale system of differential equations. In the paper of Desroches et al. (2012), one may find information about MMOs near a singular Hopf bifurcation, near a folded node, etc., analyzed from the perspective of singular perturbation theory and blow-up methods (see the book written by Dumortier 1993 for theoretical backgrounds). Most of the research in this area is made for autonomous systems, but there exist some results for non-autonomous systems as well (e.g., Szmolyan and Wechselberger 2004). In what follows we focus on the system presented in the paper

Communicated by Cristina Turner.

E. Kutafina $(\bowtie)$

Hasselt University, Agoralaan-Gebouw D, 3590 Diepenbeek, Belgium

e-mail: ekaterina.kutafina@gmail.com

E. Kutafina

AGH University of Science and Technology, Al. Mickiewicza 30, 30-059 Kraków, Poland 
of Shimizu et al. (2011)

$$
\left\{\begin{array}{l}
\epsilon \dot{x}=y+x-x^{3} \\
\dot{y}=-x-k_{1} y+B_{0}+B_{1} \sin \omega t .
\end{array}\right.
$$

This system could be seen as one of the versions of the forced van der Pol oscillator. The great advantage of this model is that it represents a simple electric circuit, so experimental results are easily available. The small parameter $\epsilon>0$ is responsible for a singular perturbation, while $B_{1}$ is also assumed to be small and positive and controls a regular non-autonomous periodic perturbation. For $B_{1}=0$ system, (1) is a two-dimensional autonomous system with classical canard explosion (see Krupa and Szmolyan 2001) controlled by the parameter $B_{0}$. The second parameter $k_{1}$ makes enough room for a generalized Hopf bifurcation (see the book of Kuznetsov 2004) in the planar system. This bifurcation (also known as Bautin bifurcation) takes place when classical Hopf bifurcation degenerates due to the vanishing first Lyapunov index. Using MATCONT, a continuation package for Matlab (see Dhooge et al. 2003), we may easily compute that Bautin bifurcation happens at $k_{1} \approx 0.513$ and $B_{0} \approx 0.365$. The diagram presented by Shimizu et al. (2011) shows that for $k_{1}=0.9 \mathrm{a}$ subcritical Hopf bifurcation is observed for $B_{0} \approx 0.20543$. Additional computations made for $k_{1}=0.2$ detect a supercritical bifurcation at $B_{0} \approx 0.4945$. These samples will be useful for the numerical computations presented in the next sections.

Systems very similar to (1) were widely studied since almost the beginning of the previous century (see van der Pol 1920). In the papers by Guckenheimer (2003) and Bold et al. (2003), the results of very extended bifurcation analysis are presented together with the references to many works on the topic.

The full system (1) reveals a very rich dynamics, for instance in the paper by Shimizu et al. (2011) authors present a sequence of stable mixed mode oscillations, which seems to be different from standard Farey sequence reported, e.g., by Petrov et al. (1992). The key difference is the fact that in the system (1) we can easily find a periodic solution with more than two large oscillations in a row. In fact, it seems to be very difficult to find a MMO with only one large oscillation. Moreover, the regions of parameters where the periodic MMOs exist are relatively small. We may also observe chaotic oscillations and bursting phenomena.

The theoretical background for multi-scale non-autonomous systems is not yet well developed. One of the typical approaches is to consider a time variable as an independent one (see Szmolyan and Wechselberger 2004). Then, we can apply the theory valid for autonomous system and get better intuition about the dynamics. In this paper, we also propose to rewrite the system as an autonomous one, but (it may seem to be artificial in the beginning) we decided to introduce two new variables $z=\sin \omega t$ and $p=\cos \omega t$. This approach allows us to reveal better analogy to the known three-dimensional systems with a fold singularity and explain the presence of MMOs using the methods known from geometric singular perturbation theory.

We write down a four-dimensional system, keeping in mind an additional equation $z^{2}+$ $p^{2}=1$, which makes it in fact a locally three-dimensional:

$$
\left\{\begin{array}{l}
\epsilon \dot{x}=y+x-x^{3} \\
\dot{y}=-x-k_{1} y+B_{0}+B_{1} z \\
\dot{p}=-\omega z \\
\dot{z}=\omega p .
\end{array}\right.
$$

Remark 1 For the further analysis, it is convenient to see the system (2) as a three-dimensional system in the space $(x, y, z)$, but due to the trigonometrical origin of $z$ there are two different $p$ values in each fixed point, thus two different values of $\dot{z}$ (difference is only in the sign). This 
means that at each fixed $(x, y, z)$ there intersect two trajectories-one following increasing values of $z$ and another one following decreasing values. In figures, it is sometimes better to show the trajectories in $(x, y, p)$ space, the small oscillations are better visible this way.

For $\epsilon=0$ system (2) has a slow manifold given by a surface $y=-x+x^{3}$ which has folds at $x= \pm \frac{\sqrt{3}}{3}$. Fenichel's theory (see Jones et al. 1995 for details) covers the dynamics of the perturbed system near normal hyperbolic points of the slow manifold. In case of the system (2) normal hyperbolicity condition fails near the folds. It occurs that the dynamics reported by Shimizu et al. (2011) shows many similarities to the prototypical three-scale model with a folded saddle-node singularity of the type II presented by Krupa et al. (2008):

$$
\left\{\begin{array}{l}
\epsilon \dot{v}=-z+f_{2} v^{2}+f_{3} v^{3} \\
\dot{z}=v-w \\
\dot{w}=\epsilon\left(\mu-g_{1} z\right) .
\end{array}\right.
$$

The generalized Bonhoeffer-van der Pol system investigated by Sekikawa et al. (2010)

$$
\left\{\begin{array}{l}
\epsilon \dot{x}=x\left(1-x^{2}\right)+y+z \\
\dot{y}=-x-k_{1} y+B_{0} \\
\dot{z}=k_{3}\left(-x-k_{1} z+B_{0}\right)
\end{array}\right.
$$

is in fact even closer to (1) and it was shown by De Maesschalck et al. (2013) (preprint) that under certain conditions it can be treated as a three time scale system. In this paper, we skip the full formal analysis of the system (2). Instead, our intention is to show what parts of the investigation could be done analogously to the papers of Krupa et al. (2008) or De Maesschalck et al. (2013). and what is different and requires a new approach. We assume that the parameters are not essentially different from those used in the paper of Shimizu et al. (2011), otherwise the behavior of the system could change drastically (see Sect. 6).

In the following sections, we start with a change of coordinates which move the right fold of the slow manifold to the origin and brings the system (2) as close as possible to the form of (3). Then, we project the flow on a slow manifold and check what kind of folded singularities is present. We discover that for certain values of bifurcation parameter $\mu$ the system can have a folded saddle-node. Rescaling of the coordinates makes the dynamics near the fold more clear. We show, that analogously to the results of De Maesschalck et al. (2013) it is possible to consider the flow near the fold as a perturbation of the certain integrable system. Skipping the other blow-up charts (or exit and entrance areas, according to Krupa et al. 2008) we make some calculations to approximate a return mechanism. Our analysis is essentially based on the theory built by Krupa et al. (2008), so to keep our paper short we did not repeat the information given there.

\section{Transition to a standard form}

It is convenient to transform the variables of the system (2) in the way that the right fold of the slow manifold is moved to the origin and is placed in a local minimum (see De Maesschalck et al. 2013 (preprint)). Thus, we make a following change of coordinates:

$$
\begin{aligned}
& x=-X+\frac{\sqrt{3}}{3}, \quad y=Y-\frac{2}{9} \sqrt{3}, \quad p=\frac{P}{B_{1}}, \\
& z=\frac{Z}{B_{1}}+\frac{3 \sqrt{3}-2 k_{1} \sqrt{3}-9 B_{0}}{9 B_{1}} .
\end{aligned}
$$


If we additionally introduce a new parameter $\mu=-\frac{3 \sqrt{3}-2 k_{1} \sqrt{3}-9 B_{0}}{9}$ then (2) takes form

$$
\left\{\begin{array}{l}
\epsilon \dot{X}=-Y+\sqrt{3} X^{2}-X^{3} \\
\dot{Y}=X-k_{1} Y+Z \\
\dot{P}=\omega(\mu-Z) \\
\dot{Z}=\omega P .
\end{array}\right.
$$

The parameter $B_{1}$ seems to disappear from the system, but in fact it is hidden in the additional relation $(Z-\mu)^{2}+P^{2}=B_{1}^{2}$. The first two equations of (6) are very similar to the ones from the system investigated by De Maesschalck et al. (2013) (after some transformations). The parameter $\omega$ in the paper of Shimizu et al. (2011) is a main bifurcation parameter and based on that results we may assume that it belongs to the interval $(0,1)$. Numerical results indicate that fixing $\omega$ and taking $\mu$ as a bifurcation parameter is possible and moreover, we can take $\omega$ small enough so the system (6) can be treated as a three time scale system. To be more precise we assume $\omega=O(\epsilon)$.

What is clearly completely different from the systems (3) and (4) is the return mechanism: in the system (3), for instance, $w$ grows as long as $\mu$ is positive and $z$ is small enough. In (6) both $Z$ and $P$ are in fact periodic functions. The domain of $P$ is the interval $\left[-B_{1}, B_{1}\right]$, while the domain of the variable $Z$ depends on the parameter $\mu: Z \in\left[-B_{1}+\mu, B_{1}+\mu\right]$. Geometrically speaking we observe the trajectories "reflecting" from the ends of the domain. No change of direction with respect to $Z$ or $P$ is possible elswhere. Another difference is a lack of stationary points for (6). If we solve the equations for a stationary points we obtain that $P=0$ together with $Z=\mu$ which imply $B_{1}=0$ and no perturbation. Instead of a singular point we obtain a periodic solution. For the same reason there is no Hopf bifurcation as such.

In Fig. 1, we present some typical solutions of (6) for $\epsilon=\omega=0.1, B_{1}=0.01, k_{1}=0.9$ and changing $B_{0}$. Note that $\mu=0$ corresponds to $B_{0} \approx 0.231$. In Fig. 1a, we see the starting periodic solution with a small amplitude in $X$ and $Y$, it corresponds to a stable stationary point in (3). With the growing $|\mu|$ this attracting periodic orbit disappears and we observe attracting MMOs. In the next pictures, we observe how the shape of mixed mode oscillations is changing with $B_{0}$. First, small oscillations after reflection make almost the same way back (Fig. 1b), then the change from small oscillations to large happens earlier and earlier until in Fig. 1e we observe no more reflection, just several small oscillations before the jump. This situations correspond to the one studied by Krupa et al. (2008). In this paper, there is an assumption, that the number of small oscillation should not be too big, otherwise the approximations are not valid anymore. On the last figure, we can see a trajectory corresponding to a relaxation oscillation in the paper of Krupa et al. (2008). It was shown in the paper of Szmolyan and Wechselberger (2004) that this trajectory lies on the torus.

These pictures raise a natural question of what is happening between the small cycle and the first observed MMO. In (3) there exists a parameter range where we may observe a small stable cycle born in the singular Hopf bifurcation (see Guckenheimer 2008), this cycle grows and undergoes multiple series of the period doubling cascades which lead to MMOs. In the paper by Petrov et al. (1992), one can find a bifurcation diagram with so-called isolas corresponding to different patterns of MMOs. In case of the system (6) instead of a small cycle we would expect a small torus. Near $k_{1}=0.9$ such a solution is difficult to detect. The reason is that as we stated before the planar Hopf bifurcation is subcritical for this value of $k_{1}$. Thus, planar small cycles are unstable and in the full system the possible tori most probably have saddle properties. So, to prove our hypothesis numerically we change the value of $k_{1}$ 


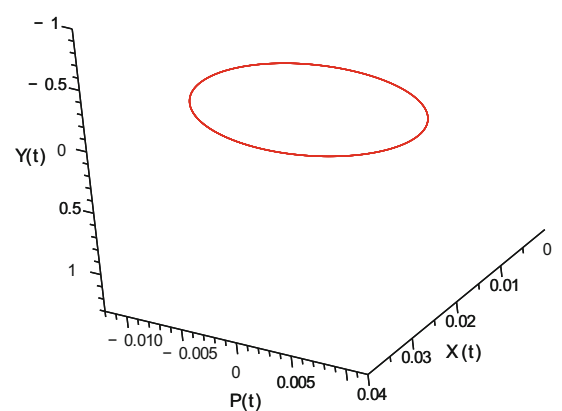

(a)

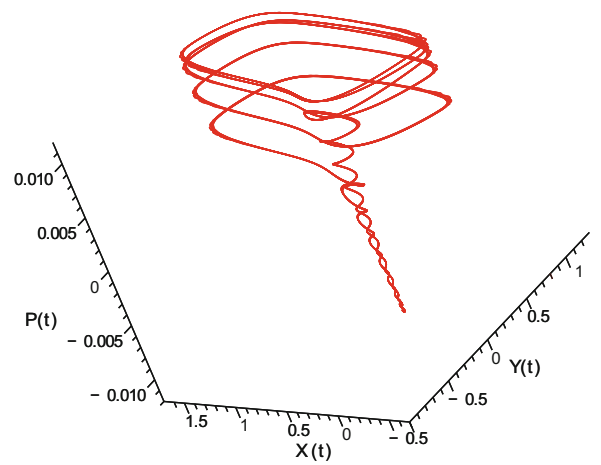

(c)

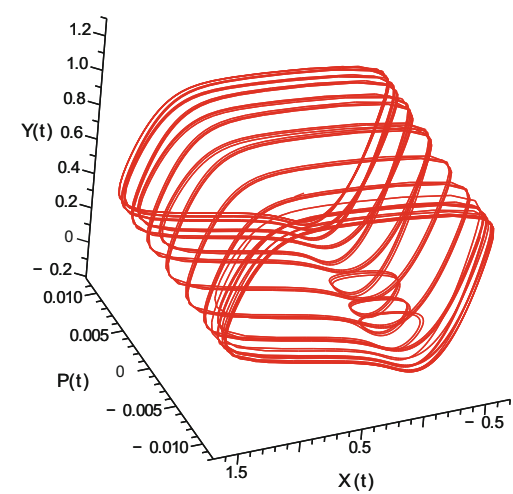

(e)

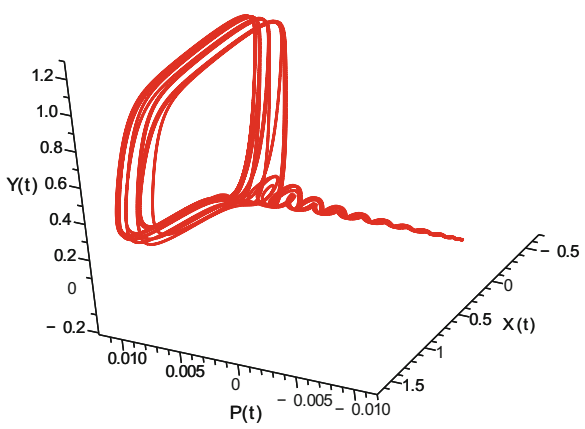

(b)

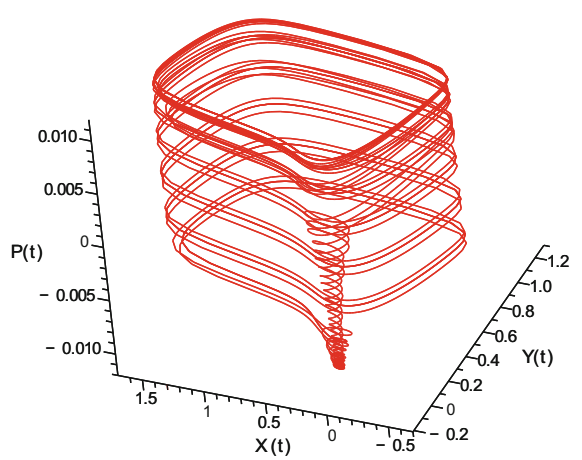

(d)

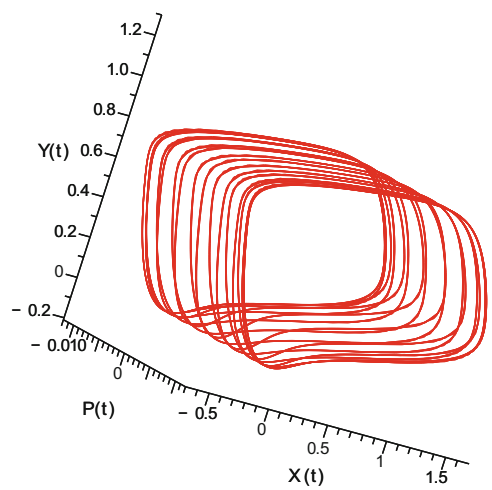

(f)

Fig. 1 Evolution from a small cycle to relaxation oscillations for changing $B_{0} . \epsilon=\omega=B_{1}=0.1, k_{1}=0.9$ and a $B_{0}=0.212$, b $B_{0}=0.206$, c $B_{0}=0.205$, d $B_{0}=0.203$, e $B_{0}=0.201$, f $B_{0}=0.200$

to 0.2 (which corresponds to a supercritical Hopf bifurcation) and indeed for $B_{0}=0.4945$ ( $\mu \approx-0.0058$ ) we observe the small torus presented on Fig. 2.

Let us now try to understand the mechanisms which form mixed mode oscillations with small number of SAOs. In Fig. 3, we present a planar projection of a singular manifold for 
Fig. 2 Small torus.

$\epsilon=\omega=B_{1}=0.1$,

$k_{1}=0.2, B_{0}=0.4945$,

$\mu \approx-0.0058$

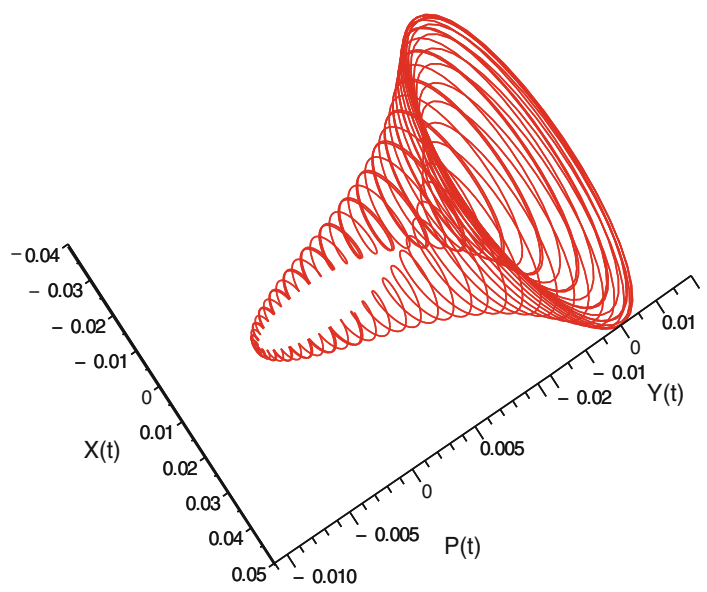

Fig. 3 Singular manifold of the system (6). Blue and red lines represent, respectively, attractive and repelling parts

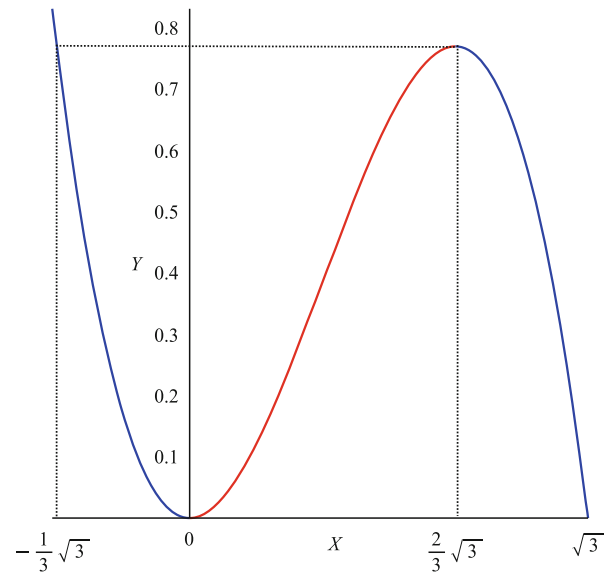

the unperturbed system (6) $(\epsilon=0)$. It consists of left and right attracting parts $\left(S_{0}^{a-}\right.$ and $\left.S_{0}^{a+}\right)$ and in the middle there is a repelling part $\left(S_{0}^{r}\right)$. When some perturbation is present $(\epsilon>0)$ these three parts perturb, respectively, into $S_{\epsilon}^{a-}, S_{\epsilon}^{a+}$ and $S_{\epsilon}^{r}$. Outside the fold regions the perturbed manifolds "inherit" the dynamics from the unperturbed system following the rules of Fenichel's theory. The neighborhood of the fold requires special analysis: we will show the presence of fold singularities and use a rescaling of the variables to understand the dynamics near $Z=0$.

In the paper by Krupa et al. (2008) (among other results) there is a very detailed analysis of the MMO's of the type $1^{k}$ (one large oscillation followed by $k$ small). The intervals of the parameter such as $1^{k}$ solution exist and is attracting are approximated. It is done by constructing a two-dimensional Poincaré map defined on the plane $v=0$. This plane contains the fold and intersects an unperturbed slow manifold transversally. Poincaré map is divided into four parts: fold area, return mechanism and transitions between fold area and return mechanism. The key simplification of the problem is the fact that the two-dimensional map can be successfully approximated by a one-dimensional map. The reason is that each trajectory jumping off the left fold is getting attracted to the right attracting part of the slow 
manifold $\left(S_{\epsilon}^{a+}\right)$, then following the perturbed flow reaches the right fold region, then jumps off again to follow a fast flow back to the left attracting branch $\left(S_{\epsilon}^{a-}\right)$. Again, following the slow flow the trajectory gets exponentially close to the straight line $C_{\epsilon}^{-}$which denotes the intersection of $S_{\epsilon}^{a+}$ and the plane $v=0$. As a result, it is possible to study a one-dimensional Poincaré map $C_{\epsilon}^{-} \rightarrow C_{\epsilon}^{-}$, which is a much easier task. The line $C_{\epsilon}^{-}$contains a critical canard point $w=w_{c r}$ which corresponds to the transversal intersection of the left attracting manifold $S_{\epsilon}^{a-}$ and the repelling manifold $S_{\epsilon}^{r}$. Every trajectory starting from the point on $C_{\epsilon}^{-}$ with $w$ coordinate smaller that $w_{c r}$ undergoes small oscillations, while if it starts on the other side it jumps to $S_{\epsilon}^{a+}$ as described before following the fast flow first and then creating a large loop (we call it return mechanism). It is possible to define sectors of rotation $R S^{k}$-intervals of $C_{\epsilon}^{-}$such as if the trajectory starts in $R S^{k}$ it undergoes exactly $k$ small oscillations before leaving the fold region. Precise analysis of the dynamics near the fold is only possible for $w$ small enough. In that case, the solutions can be approximated as perturbations of a certain integrable two-dimensional system.

Our numerical and analytical observations indicate that it should be possible to perform an analogous reduction for the system (6). As it was mentioned before, our current goal is just to indicate some similarities and differences as well as to prepare a background for a further detailed analysis.

\section{Folded singularities}

Let us start our analysis with the examination of the folded singularities of the system (6). To do so we project system (6) on the slow manifold $Y=\sqrt{3} X^{2}-X^{3}=f(X)$. De-singularized (see Desroches et al. 2012) system has the following form:

$$
\left\{\begin{array}{l}
X^{\prime}=-X-k_{1} f(X)+Z \\
P^{\prime}=\omega f^{\prime}(X)(\mu-Z) \\
Z^{\prime}=\omega f^{\prime}(X) P
\end{array}\right.
$$

Here, ()$^{\prime}$ in the RHS denotes the rescaled time-derivative $\left(\dot{)} f^{\prime}(X)\right.$. Time flow changes the directory for $X$ such as $f^{\prime}(X)<0$, so on the attracting branches of the manifold.

Solving the equations for the stationary points, we obtain $\left(0, \pm \sqrt{B_{1}^{2}-\mu^{2}}, 0\right)$ on the left fold and $\left(\frac{2}{3} \sqrt{3}, P_{0}, \frac{2}{3} \sqrt{3}\left(\frac{2}{3} k_{1}-1\right)\right)$ on the right fold. $P_{0}$ denotes a value of $P$ corresponding to $Z=\frac{2}{3} \sqrt{3}\left(\frac{2}{3} k_{1}-1\right)$. For the considered values of the parameters: $k_{1} \in(0,1), B_{1}$ and $\mu$ small there are no singularities on the right fold. Assuming $|\mu|<B_{1}>0$ we have two stationary points $A_{1}=\left(0, \sqrt{B_{1}^{2}-\mu^{2}}, 0\right)$ and $A_{2}=\left(0,-\sqrt{B_{1}^{2}-\mu^{2}}, 0\right)$ on the left fold. Corresponding eigenvalues are:

$$
\lambda_{1,2,3}\left(A_{1}\right)=\left(0, \frac{1}{2} \pm \frac{1}{2} \sqrt{1-8 \sqrt{3} \omega \sqrt{B_{1}^{2}-\mu^{2}}}\right)
$$

and

$$
\lambda_{1,2,3}\left(A_{2}\right)=\left(0, \frac{1}{2} \pm \frac{1}{2} \sqrt{1+8 \sqrt{3} \omega \sqrt{B_{1}^{2}-\mu^{2}}}\right) .
$$

In both points zero eigenvalue corresponds to $P$-direction. Assuming $B_{1}$ and $\mu$ small enough the first point is a node while the second one is a saddle in the $(X, Z)$ space. For $\mu= \pm B_{1}$ or $\omega \rightarrow 0$ two singular points melt into a single one placed in the origin and it is a folded 
saddle-node (in $(X, Z)$ space again) with the eigenvector corresponding to zero eigenvalue transversal to a fold. In case of the system (3) we have only one singular point and the saddlenode appears as folded saddle bifurcating into a folded node. However in the analyzed range of parameters singularity moves outside the assumed domain $(w \sim O(\epsilon))$. The same situation is present in the system (6) for $|\mu|>B_{1}$ (which seems to be satisfied for the parameter range where we observe MMOs with a small number of SAOs). Krupa and Wechselberger (2010) investigate the folded saddle-node singularity in the domain wide enough to take into account the singularities but in that case different phenomena play a key role.

\section{Fold region in the rescaled coordinates}

In this section, we investigate the fold area using a classical approach of geometrical theory singular perturbations. We make a rescaling which is in fact identical to considering a family chart of blown-up coordinates:

$$
X=\epsilon^{\frac{1}{2}} \bar{X}, \quad Y=\epsilon \bar{Y}, \quad P=\epsilon^{\frac{1}{2}} \bar{P}, \quad Z=\epsilon^{\frac{1}{2}} \bar{Z} .
$$

After an additional time rescaling (we keep the $(\dot{)}$ notation) the system (6) takes form

$$
\left\{\begin{array}{l}
\dot{\bar{X}}=-\bar{Y}+\sqrt{3} \bar{X}^{2}-\sqrt{\epsilon} \bar{X}^{3} \\
\dot{\bar{Y}}=\bar{X}-k_{1} \sqrt{\epsilon} \bar{Y}+\bar{Z} \\
\dot{\bar{P}}=\omega(\bar{\mu}-\sqrt{\epsilon} \bar{Z}) \\
\dot{\bar{Z}}=\omega \sqrt{\epsilon} \bar{P} .
\end{array}\right.
$$

For $\epsilon=0$ and $\bar{Z}=0$ (which is satisfied for $B_{1}$ and $\mu=0$ ), first two equations of the system (9) form an integrable system with a constant of motion given by

$$
H(\bar{X}, \bar{Y})=\frac{1}{2} e^{-2 \sqrt{3} \bar{Y}}\left(-\bar{X}^{2}+\frac{\bar{Y}}{\sqrt{3}}+\frac{1}{6}\right) .
$$

The special solution corresponding to $H(\bar{X}, \bar{Y})=0$

$$
\bar{\gamma}_{0}^{0}(t)=\left(\frac{1}{2 \sqrt{3}} t, \frac{1}{4 \sqrt{3}} t^{2}-\frac{1}{2 \sqrt{3}}\right)
$$

separates the area of periodic solutions and open level curves. This solution also corresponds to a singular canard (singular limit of intersection of the manifolds $S_{\epsilon}^{a-}$ and $S_{\epsilon}^{r}$ ).

The integrable system is exactly the same as the one examined by De Maesschalck et al. (2013). Moreover, if we assume $\omega=O(\epsilon)$ as mentioned before, we can treat the full fourdimensional system as a perturbation of the integrable two-dimensional one. The singular canard trajectory perturbs into a strong canard lying on the intersection of perturbed attractive and repelling manifolds. To compute the corresponding value of $\bar{Z}$ (we call it $\bar{Z}_{c r}$ ) we may follow the calculations from the paper by De Maesschalck et al. (2013) (the only difference is a plus sign at $\bar{Z}$ in the second equation) and conclude that the critical value of $\bar{Z}$ is given by

$$
\bar{Z}_{c r}=-\frac{\sqrt{3}\left(1+2 k_{1}\right)}{24} \sqrt{\epsilon} .
$$

The idea behind this formula is as following: $\bar{Z}_{c r}$ divides the area of almost-periodic orbits from non-periodic ones. Thus the orbit passing this critical point can be treated as 
a periodic one, but with an infinite period time. Small $\omega$ allows us to treat $\bar{P}$ and $\bar{Z}$ as constants, while small $\epsilon$ guarantees that the orbits could be approximated by the orbits of the integrable planar system. The computations are almost identical to those made by De Maesschalck et al. (2013), but their presentation would involve a lot of additional notations.

If we recall the original variables we obtain $Z_{c r}=-\frac{\sqrt{3}\left(1+2 k_{1}\right)}{24} \epsilon$. Since there is a relationship between $P$ and $Z$ we can compute $P_{c r}= \pm \sqrt{B_{1}^{2}-\left(Z_{c r}-\mu\right)^{2}}$. That means that there exist two canard trajectories (obviously if $\epsilon$ and $\mu$ are small enough both $Z_{c r}$ and $P_{c r}$ belong to the domain). We can observe this phenomena for instance on Fig. 1e: the area of jumps is followed by the small oscillations and then by jumps again. However in the system (6) this double canard occur due to the symmetry of the trigonometric functions, it could be interesting to construct a three-dimensional polynomial system with similar geometry.

\section{Return mechanism}

To obtain an approximation of the return mechanism let us go back to the projection on the slow manifold described by the system (7). For $\epsilon$ and $\omega$ small enough we may assume that the change of $P$ during "fast time" intervals can be neglected, so the slow manifold projection provides us enough information to approximate the map. If we divide the second equation of (7) by the first one we obtain

$$
\frac{\mathrm{d} P}{\mathrm{~d} X}=\omega \frac{f^{\prime}(X)(\mu-Z)}{X-k_{1} f(X)+Z} .
$$

We assume that $Z$ is small comparing to $X-k_{1} f(X)$ which is justified by the domain where $Z$ is defined. Then (recalling the relationship $\left.(Z-\mu)^{2}+P^{2}=B_{1}^{2}\right)$ we may rewrite (10) as

$$
\frac{\mathrm{d} P}{ \pm \sqrt{B_{1}^{2}-P^{2}}}=\omega \frac{f^{\prime}(X) \mathrm{d} X}{X-k_{1} f(X)}
$$

where the sign at the square root depends on the direction the trajectory is following. It is + for increasing $P$ and - for decreasing. Let us introduce an auxiliary variable $W=\omega t$. To cover all possible situations it is sufficient to take $W \in[0,3 \pi]$. We can easily compute that $\mathrm{d} W=\frac{\mathrm{d} P}{ \pm \sqrt{B_{1}^{2}-P^{2}}}$, which transforms (11) into a simpler dependence

$$
\mathrm{d} W=\omega \frac{f^{\prime}(X) \mathrm{d} X}{X-k_{1} f(X)}
$$

It is clear from Fig. 3 that for the full return loop the variable $X$ needs to run from $\sqrt{3}$ to $\frac{2}{3} \sqrt{3}$ (right attractive manifold) and then from $-\frac{\sqrt{3}}{3}$ to 0 (left one).

If we denote by $P_{0}$ a start point on $S_{\epsilon}^{a-}$ close to a jump area then a corresponding $W_{0}$ is assumed to be given by $\arccos \frac{P_{0}}{B_{1}}$ if in $P_{0} P$ is a decreasing function of time and $2 \pi-\arccos \frac{P}{B_{1}}$ otherwise. We may approximate the result of the return mechanism as 


$$
\begin{aligned}
W_{1} & =W_{0}+\omega\left(\int_{\sqrt{3}}^{\frac{2}{3} \sqrt{3}} \frac{f^{\prime}(X) \mathrm{d} X}{X-k_{1} f(X)}+\int_{-\frac{\sqrt{3}}{3}}^{0} \frac{f^{\prime}(X) \mathrm{d} X}{X-k_{1} f(X)}\right) \\
& =W_{0}+3 \omega\left(1-\frac{k_{1}}{2}\right) .
\end{aligned}
$$

Now, depending on which part of the domain we landed, we have

$$
W_{1}^{-}=\arccos \frac{P_{1}}{B_{1}}, \quad W_{1}^{+}=2 \pi-\arccos \frac{P_{1}}{B_{1}} \quad \text { or } \quad W_{1}^{=}=2 \pi+\arccos \frac{P_{1}}{B_{1}} .
$$

Checking all possible combinations (the trajectory can come back without or with hitting the domain boarder) we obtain the formula

$$
P_{1}=B_{1} \cos \left(\arccos \frac{P_{0}}{B_{1}} \pm 3 \omega\left(1-\frac{k_{1}}{2}\right)\right)
$$

where the sign is - if we follow the direction of increasing $P$ and + otherwise. Numerical experiments confirm this approximation. Let us underline that this formula covers also the case when there is a "reflexion" effect in the way. The trigonometric functions are responsible for the "compression" close to the border of the domain while in the paper by Krupa et al. (2008) the distance between initial and end points of the return mechanism is approximately constant.

The formula (13) shows that the number of LAOs per length of the domain depends only on the parameters $k_{1}$ and $\omega$. This approximation is good enough for the analysis based on Krupa et al. (2008), but unfortunately does not allow us to generate periodic orbits. It is however practically easier to catch a (numerically) periodic orbit for $k_{1}=0.2$ then $k_{1}=0.9$, one of the possible reason is a clearer picture due to less LAOs per period. An example of such an orbit is presented in Fig. 4.

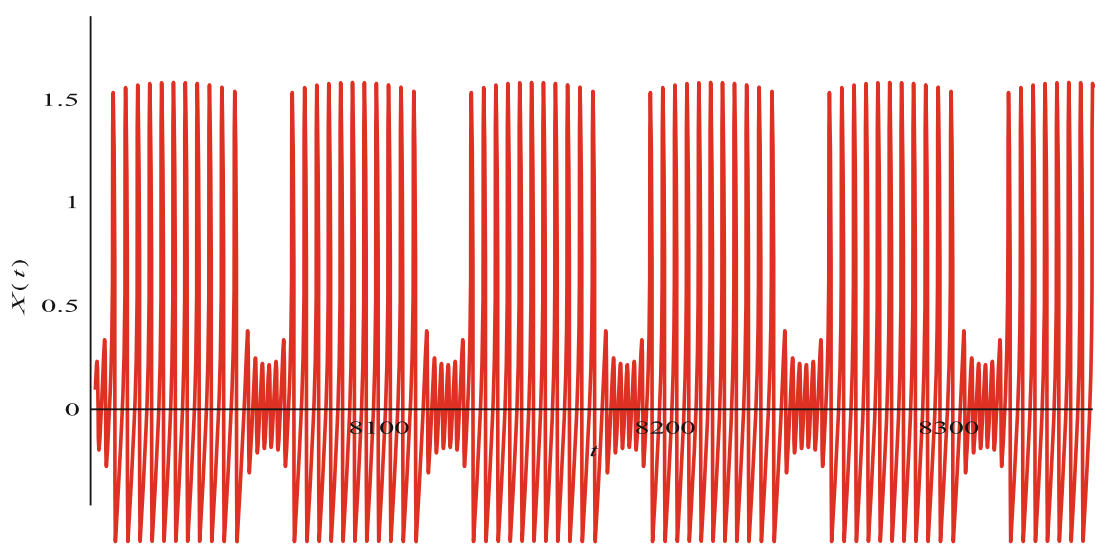

Fig. $4 \epsilon=\omega=0.1, k_{1}=0.2, B_{1}=0.01, B_{0}=0.4815, \mu \approx-0.0119$ 


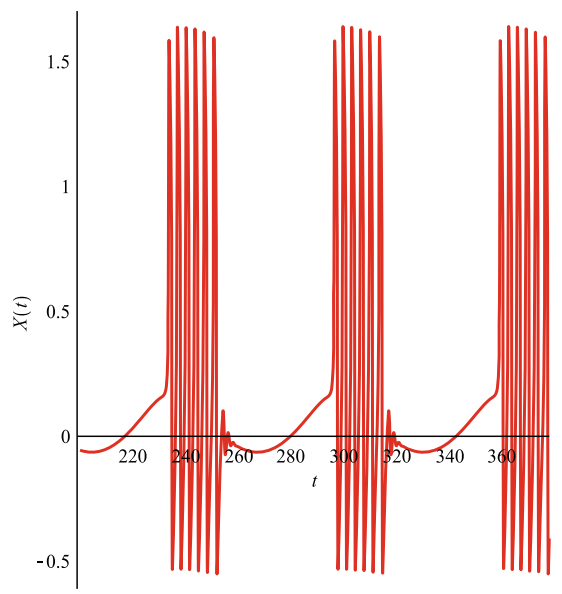

(a)

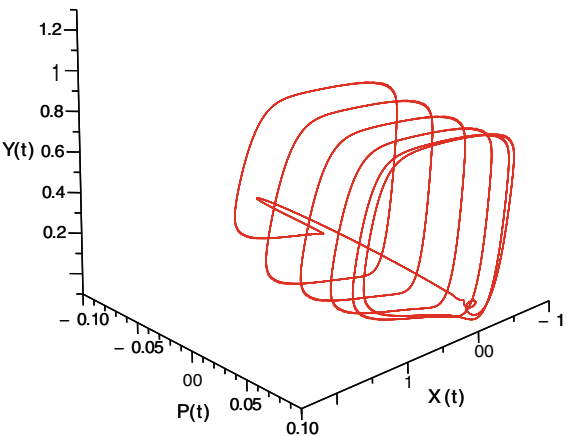

(b)

Fig. $5 \epsilon=\omega=B_{1}=0.1, k_{1}=0.9, B_{0}=0.201, \mu \approx-0.03$

\section{Discussion}

In this paper, we presented a locally three-dimensional system which is equivalent to a Bonhoeffer-van der Pol model. Keeping the parameter region close to the examples from the paper of Shimizu et al. (2011), we discussed the similarity of that system to known models with folded saddle-node II singularity. Some additional assumptions were made to separate the time scales and eliminate the effect of the stationary points. A brief analysis of the fold region and an approximation of the return mechanism were provided.

We observed several differences such as a presence of two canards next to each other and non-homogeneous distribution of the return mechanism. Basically, these effects are present due to the choice of the variables. On the other hand this choice not only draw a parallel to the known and well-analyzed systems, but also could be useful for the construction of polynomial systems with interesting dynamics.

Further analysis of the system could be especially interesting if we recall that the original system (1) models a simple circuit, which can be used for experiments with the parameters. This implies that this circuit can be a very simple physical model for the three time scale systems with a folded saddle-node singularity.

In our research, we concentrated on the indication of the parameter range where the particular type of dynamics takes place. It could be interesting to go outside this region and take a look on the nearby bifurcations based on the papers of Guckenheimer (2003) and Bold et al. (2003). To underline how different the behavior of the trajectory could be, in Fig. 5 we present the periodic MMO with the so-called bursting phenomena present. This type of solution is typical for the neuronal models (see Izhikevich 2007). The parameters are like in case of Fig. 1, except $B_{1}=0.1$. This change results that the stationary points on the fold existing even for relatively large $|\mu|$. Another interesting phenomenon to study is presence of the delayed Hopf bifurcation (see Krupa and Wechselberger 2010; Neishtadt 1987).

Acknowledgments Author is very grateful to prof. Peter De Maesschalck for many stimulating discussions. The presented research was supported by the Research Foundation Flanders (FWO) under grant number G.0939.10N and by Polish Ministry of Science and Higher Education. 
Open Access This article is distributed under the terms of the Creative Commons Attribution License which permits any use, distribution, and reproduction in any medium, provided the original author(s) and the source are credited.

\section{References}

Bold K, Edwards C, Guckenheimer J, Guharay S, Hoffman K, Hubbard J, Oliva R, Weckesser W (2003) The forced van der Pol equation II: canards in the reduced system. SIAM J Appl Dyn Syst 2:570-608

De Maesschalck P, Kutafina EV Popović N (2013) The Bonhoeffer-van der Pol system in the 3-time scale regime (submitted)

Desroches M, Guckenheimer J, Krauskopf B, Kuehn C, Osinga H, Wechselberger M (2012) Mixed-mode oscillations with multiple time scales. SIAM Rev 54(2):21

Dhooge A, Govaerts W, Kuznetsov Yu (2003) MatCont: a MATLAB package for numerical bifurcation analysis of ODEs. ACM TOMS 29:141-164

Dumortier F (1993) Techniques in the theory of local bifurcations: blow-up, normal forms, nilpotent bifurcations, singular perturbations. Kluwer, Dordrecht

Guckenheimer J (2008) Singular Hopf bifurcation in systems with two slow variables. SIAM J Appl Dyn Syst 7(4): 1355

Guckenheimer J, Hoffman K, Weckesser W (2003) The forced van der Pol equation I: the slow flow and its bifurcations. SIAM J Appl Dyn Syst 2:135

Izhikevich EN (2007) Dynamical systems in neuroscience: the geometry of excitability and bursting. Computational Neuroscience, MIT Press, Cambridge

Jones CKRT (1995) Geometric singular perturbation theory. Springer, Berlin, pp 44-118

Krupa M, Szmolyan P (2001) Relaxation oscillation and canard explosion. J Differ Equ 174:312-368

Krupa M, Wechselberger M (2010) Local analysis near a folded saddle-node singularity. J Differ Equ 248:2841-2888

Krupa M, Popovic N, Kopell N (2008) Mixed-mode oscillations in three timescale systems-a prototypical example. SIAM J Appl Dyn Syst Online 7(2):361

Kuznetsov Yu (2004)Elements of applied bifurcation theory. Springer, Berlin

Neishtadt AI (1987) Persistence of stability loss for dynamic bifurcations I. Differ Equ 23:1385-1390

Petrov V, Scott SK, Showalter K (1992) J Chem Phys 97:6191

Sekikawa M, Inaba N, Yoshinaga T, Hikihara T (2010) Period-doubling cascades of canards from the extended Bonhoeffervan der Pol oscillator. Phys Lett A 374:3745-3751

Shimizu K, Sekikawa M, Inaba N (2011) Mixed-mode oscillations and chaos from a simple second-order oscillator under weak periodic perturbation. Phys Lett A 375:1566

Szmolyan P, Wechselberger M (2004) Relaxation Oscillations in $R^{3}$. J Differ Equ 200:69-104

van der Pol B (1920) A theory of the amplitude of free and forced triode vibrations. Radio Rev 1:701-710 $(754-762)$ 